LA-UR- 96-2103

CONF-9605/94 -1

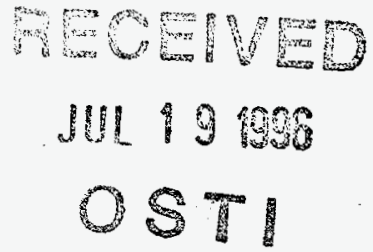

$\mathrm{Nu} \mathrm{Xu}$ for the NA44 Collaboration

Author(s):

Submitted to:

Strangeness '96, Budapest, Hungary, May 15-17, 1996

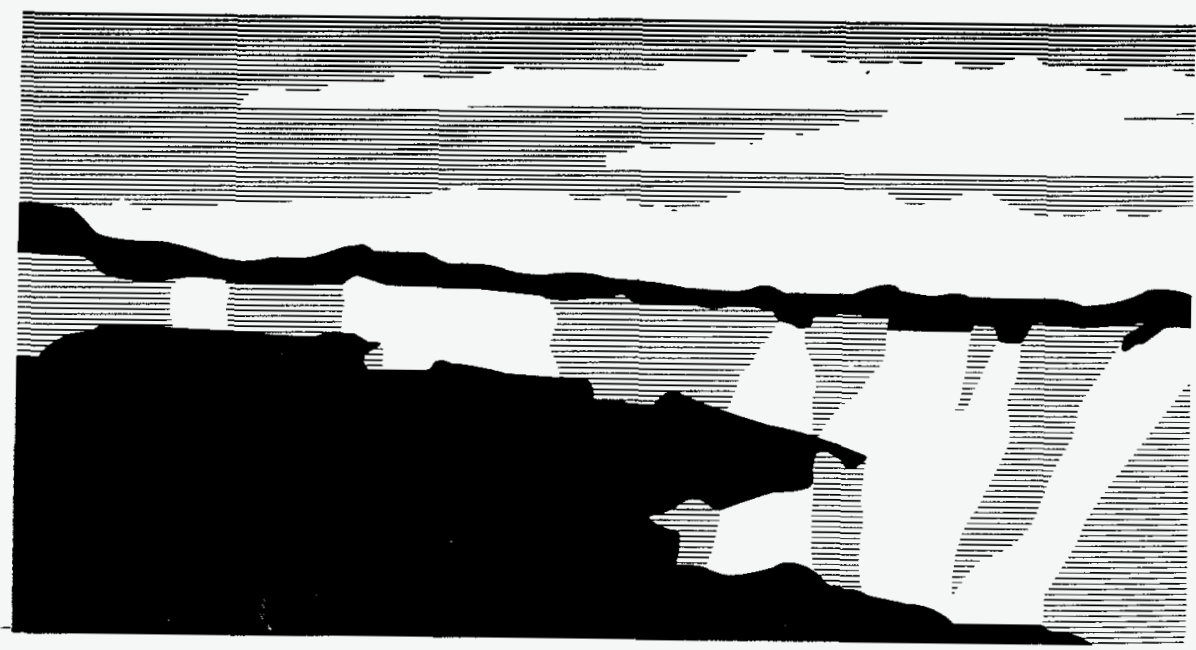

Los Alamos National Laboratory, an affirmative action/equal opportunity emplóyer, is operated by the University of California for the U.S. Department of Energy under contract W-7405-ENG-36. By acceptance of this article, the publisher recognizes that the U.S. Government retains a nonexclusive, royalty-free license to publish or reproduce the published form of this contribution, or to allow others to do so, for U.S. Government purposes. The Los Alamos National Laboratory requests that the publisher identify this article as work performed under the auspices of the U.S. Department of Energy.

I.G. Bearden, H. Bøggild, J. Boissevain, J. Dodd, B. Erazmus, S. Esumi, C.W. Fabjan, D. Ferenc, D. E. Fields, A. Franz, J. Gaardhøje, O. Hansen, D. Hardtke, H. van Hecke, E.B. Holzer, T. Humanic, P. Hummel, B.V. Jacak, R. Jayanti, M. Kaneta, M. Kopytine, M. Leltchouk, T. Ljubicic B. Lörstad, N. Maeda, A. Medvedev, M. Murray, S. Nishimura, H. Ohnishi, G. Paic, S.U. Pandey, F. Piuz, J. Pluta, V. Polychronakos, M. Potekhin, G. Poulard, A. Sakaguchi, J. Simon-Gillo, J. Schmidt-Sørensen, W. Sondheim, M. Spegel, T. Sugitate, J. P. Sullivan, Y. Sumi, W.J. Willis K.Wolf, N. Xu, and D.S. Zachary

NBI-LANL-Columbia-CERN-Nantes-Hiroshima-Zagreb-OSU-Lund-Texas A\&M-BNL 


\section{DISCLAIMER}

Portions of this document may be illegible in electronic image products. Images are produced from the best available original document. 


\title{
FREEZE-OUT CONDITIONS IN ULTRARELATIVISTIC HEAVY-ION COLLISIONS
}

\author{
Nu Xu for the NA44 Collaboration \\ Los Alamos National Laboratory, Los Alamos, NM 87545, U.S.A.
}

I.G. Bearden, H. Bøggild, J. Boissevain, J. Dodd, B. Erazmus, S. Esumi, C.W. Fabjan, D. Ferenc, D. E. Fields, A. Franz, J. Gaardhøje, O. Hansen, D. Hardtke, H. van Hecke, E.B. Holzer, T. Humanic, P. Hummel, B.V. Jacak, R. Jayanti, M. Kaneta, M. Kopytine, M. Leltchouk, T. Ljubicic, B. Lörstad, N. Maeda, A. Medvedev, M. Murray, S. Nishimura, H. Ohnishi, G. Paic, S.U. Pandey, F. Piuz, J. Pluta, V. Polychronakos, M. Potekhin, G. Poulard, A. Sakaguchi, J. Simon-Gillo, J. Schmidt-Sørensen, W. Sondheim, M. Spegel, T. Sugitate, J. P. Sullivan, Y. Sumi, W.J. Willis, K.Wolf, N. Xu, and D.S. Zachary

NBI-LANL-Columbia-CERN-Nantes-Hiroshima-Zagreb-OSU-Lund-Texas A\&M-BNL

\begin{abstract}
We present recent results on single particle transverse momentum distributions of pions, kaons, and protons, measured in CERN Experiment $\mathrm{NA} 44$, of $200 \mathrm{~A} \cdot \mathrm{GeV} / \mathrm{c} \mathrm{S}+\mathrm{S}$ and $158 \mathrm{~A} \cdot \mathrm{GeV} / \mathrm{c} \mathrm{Pb}+\mathrm{Pb}$ central collisions. By comparing these data with thermal and transport models, freeze-out parameters like the temperature $T_{f o}$ and the chemical potentials $\left(\mu_{q}, \mu_{s}\right)$ are extracted and discussed.
\end{abstract}

\section{Introduction}

The physics motivation of ultrarelativistic heavy-ion collisions is to prepare nuclear matter with high baryon and energy densities, and hope to reach a new form of matter called quark-gluon plasma. By studying the decay of the system, we will gain knowledge of strong interactions under these extreme conditions and shed light on the puzzle of quark confinement.

The fireball created in these collisions contains a finite number of particles and its life time and spatial size are also limited. Furthermore, due to the finite interaction cross section among hadrons, statistically the freeze-out occurs from the outside toward the inside of the fireball. One may imagine that free streaming hadrons are evaporated from the fireball surface. Therefore it is important to understand if the final hadron spectra are those of a thermalized system, and if the $u, d$, and $s$ quark distributions follow the statistical weights. This has been a subject of interest for many years, see refs. [1]-[7]. In this paper, after a brief description of the NA44 experiment, we will discuss the transverse momentum distributions of pions, kaons, and protons from $200 \mathrm{~A} \cdot \mathrm{GeV} / \mathrm{c} \mathrm{S}+\mathrm{S}$ and $158 \mathrm{~A} \cdot \mathrm{GeV} / \mathrm{c} \mathrm{Pb}+\mathrm{Pb}$ central collisions. From this study, the freeze-out temperature parameter $T_{f o}$ for ultrarelativistic heavy-ion collisions is established. In order to gain insight into the chemical properties and the possible medium effects [8] in the produced fireball, 
we then study particle ratios to extract the $u(d)$ and $s$ quark chemical potential parameters $\left(\mu_{q}, \mu_{s}\right)$.

\section{CERN Experiment NA44}

The NA44 spectrometer is designed to measure one- and two-particle distributions of charged hadrons near the center of mass rapidity $\left(y_{m i d} \approx 3\right)$ over a transverse momentum range of $0 \leq p_{T} \leq 1.6 \mathrm{GeV} / \mathrm{c}$. Threshold Cherenkov counters and timeof-flight (TOF) scintillator hodoscopes are used for particle identification and momentum reconstruction. The TOF resolution is about $100 \mathrm{ps}$ and momentum spread is about $\delta p / p \approx 0.5 \%$. The interaction trigger is provided by two scintillator paddles placed downstream of the target. For AA collisions, the trigger cross section is approximately $\sigma_{\text {trig }} / \sigma_{\text {geom }} \approx 10 \%$, while for $\mathrm{pA}$ collisions a minimum bias trigger was used. More details of the spectrometer can be found elsewhere $[9,10]$.

The energy of the proton beam is $450 \mathrm{GeV}, y_{N N}=3.44$; for the Sulphur beam, it is $200 \mathrm{~A} \cdot \mathrm{GeV}$ with $y_{N N}=3.03$; and for the lead beam $158 \mathrm{~A} \cdot \mathrm{GeV}$ with $y_{N N}=2.91$. In the following discussions, we will focus on the $8 \mathrm{GeV} / \mathrm{c}$ momentum setting. Here the spectrometer have rapidity span of $(2.75-2.95)$ and $(2.6-2.8)$ for kaons and protons, respectively. In order to use pions from the mid-rapidity region, low $p_{T}$ part of pion data was taken from the $4 \mathrm{GeV} / \mathrm{c}$ setting and high $p_{T}$ part was taken from the $8 \mathrm{GeV} / \mathrm{c}$ setting.

\section{Results and Discussion}

In heavy-ion physics, particle distributions are often shown in two directions: the longitudinal direction which is along the beam $(z)$ direction and the transverse direction, perpendicular to the beam direction. The variable used for the longitudinal distribution is rapidity $y=0.5 \log \left(E+p_{z}\right) /\left(E-p_{z}\right)$ and the variable used for transverse distributions is either the transverse momentum $p_{T}=\left(p_{x}^{2}+p_{y}^{2}\right)^{1 / 2}$ or transverse kinetic energy $k_{T}=m_{T}-$ mass. $^{1}$

Proton rapidity distributions [11] from the $\mathrm{Pb}+\mathrm{Pb}$ collisions show a rather large number of protons piled up around mid-rapidity, implying, that even at the CERN energy, a high degree of stopping is achieved. The strong Coulomb effect [12, 13] observed in the $\mathrm{Pb}+\mathrm{Pb}$ collisions provides additional evidence of high nuclear stopping.

At the high $p_{T}$ limit, a transverse kinetic energy distribution can be represented by a simple exponential function: $\exp \left(-m_{T} / T\right) .{ }^{2}$ Here $T$ is the slope parameter. The magnitude of the slope parameter can provide information about collective transverse flow, and deviations from the exponential distribution may signal new physics like the low $p_{T}$ enhancement, medium effects [8] etc.

\footnotetext{
${ }^{1}$ Unless otherwise noted $\hbar=c=1$.

${ }^{2}$ Note that the exponential shape is only consistent with a thermal distribution, but not necessarily a proof of thermalization.
}

\section{DISCLAIMER}

This report was prepared as an account of work sponsored by an agency of the United States Government. Neither the United States Government nor any agency thereof, nor any of their employees, makes any warranty, express or implied, or assumes any legal liability or responsibility for the accuracy, completeness, or usefulness of any information, apparatus, product, or process disclosed, or represents that its use would not infringe privately owned rights. Reference herein to any specific commercial product, process, or service by trade name, trademark, manufacturer, or otherwise does not necessarily constitute or imply its endorsement, recommendation, or favoring by the United States Government or any agency thereof. The views and opinions of authors expressed herein do not necessarily state or reflect those of the United States Government or any agency thereof. 


\subsection{Transverse Momentum Distributions}

Figure 1 shows the transverse kinetic energy distributions of charged pions, kaons, and protons. The dashed lines represent an exponential fit to the distributions and the fitted slope parameters are indicated in the figure. ${ }^{3}$ First of all, the exponential functions fit the data well except for pions in the low $p_{T}$ region, where the contributions of resonance decays become important. Secondly, the slope parameters increase as particle mass increases and this is independent of the sign of the particles. To make this point clearer, we summarize the slope parameters of pions, kaons, and protons from three collision systems: $\mathrm{p}+\mathrm{p}[14](\sqrt{s} \approx 23 \mathrm{GeV})$, $\mathrm{S}+\mathrm{S}(\sqrt{s} \approx 19.4 \mathrm{~A} \cdot \mathrm{GeV})$, and $\mathrm{Pb}+\mathrm{Pb}(\sqrt{s} \approx 17.3 \mathrm{~A} \cdot \mathrm{GeV})$ in Fig. 2 where the slope parameters $T$ are plotted against particle mass.

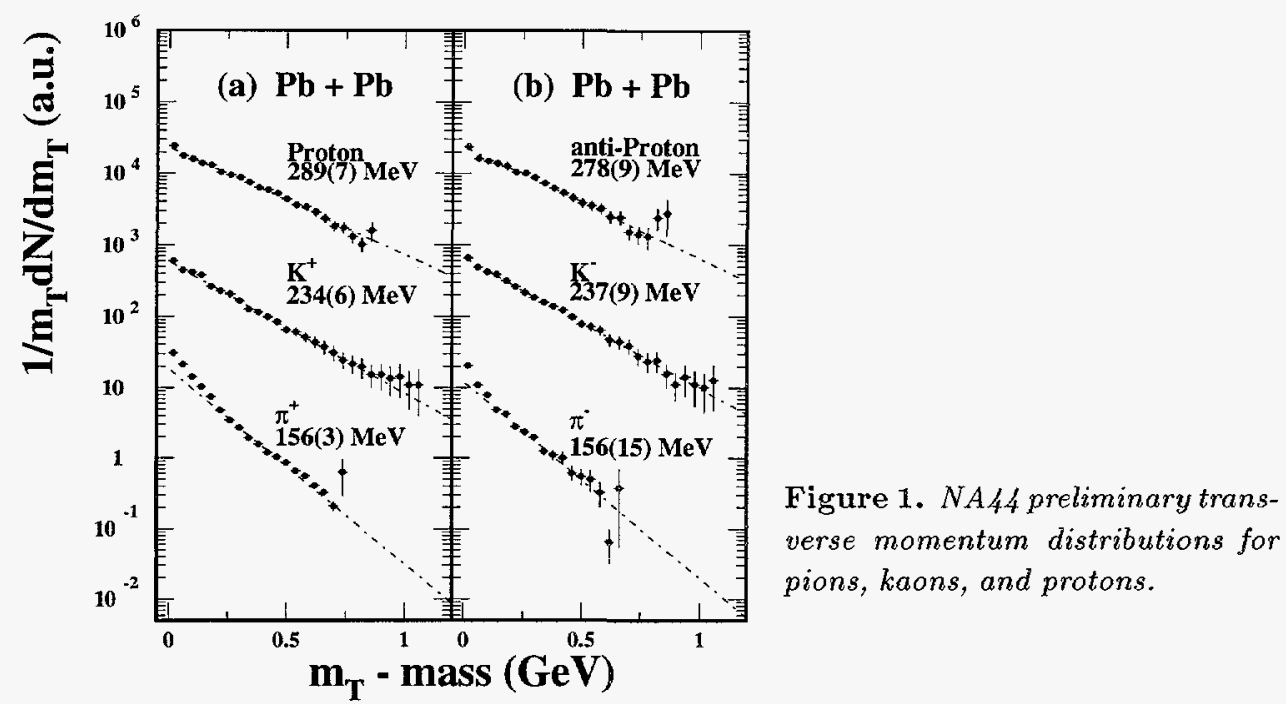

A distinct difference between the elementary $\mathrm{p}+\mathrm{p}$ collision and heavy-ion collisions can be seen in the slope parameters. It is also interesting to observe that all curves converge to a point about $T_{f o} \approx 145 \pm 15 \mathrm{MeV}$ at the limit mass $\rightarrow 0$ as indicated by the shaded bars in the plot. In hydrodynamics, matter flows, i.e., all particles flow at a same collective velocity. Classically, the collective kinetic energy will then depend on the particle mass: particles with higher mass will carry higher energy. Therefore the slope parameter is a measure of the energy of the transverse motion. The transverse motion contains both thermal (random) and collective modes. The intrinsic freeze-out temperature is only determined by the thermal motion.

For $\mathrm{p}+\mathrm{p}$ collisions, due to the saturation of the phase-space, the final hadron distributions show characteristic of thermalization $[1,15,16]$. Thus the slope pa-

\footnotetext{
${ }^{3}$ To avoid the distortion caused by high mass resonances in the pion spectra, the fit is for $k_{T}$ $\geq 0.2 \mathrm{GeV}$.
} 
rameter may reflects the true freeze-out temperature. This is supported by the fact that the slope parameters of pion, kaon and proton are very similar, around $150 \mathrm{MeV}$. However, for heavy-ion collisions, rescattering becomes more and more important and a collective transverse motion gradually develops. The slopes in the T-mass plot indeed demonstrate such characteristic hydrodynamic behavior. Furthermore, as one can see from the figure, the heavier the colliding system, the steeper the slope in the plot.

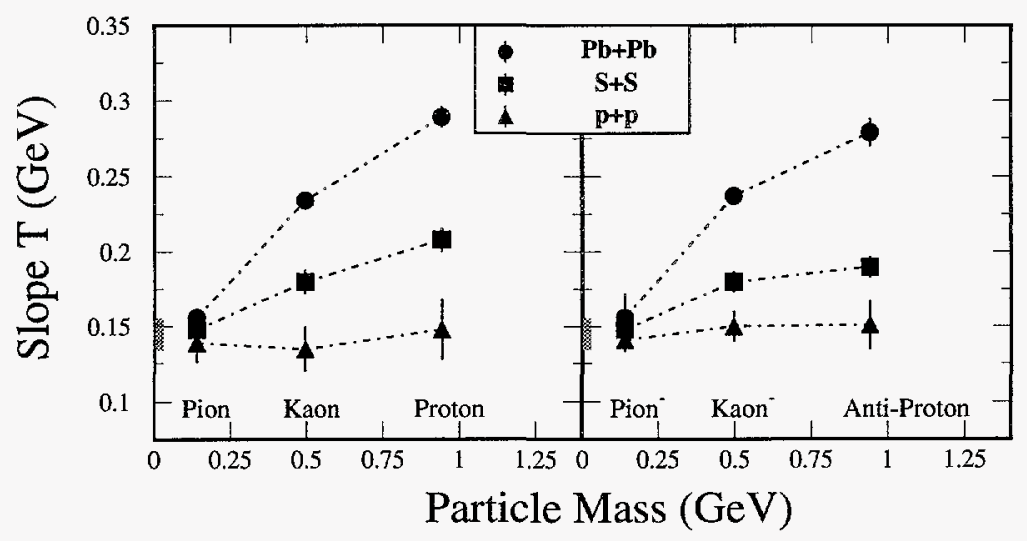

Figure 2. Slope parameter $T$ as a function of particle mass for $p+p[14], S+S$, and $P b+P b$ central collisions. The AA data are preliminary.

It is worth noting that very similar behavior can be found at the AGS energies $\left(E_{\text {beam }} \approx 10-15 \mathrm{~A} \cdot \mathrm{GeV}\right)$. The characteristic freeze-out temperature $T_{f o}$ is also about $150 \mathrm{MeV}$ ! On the other hand, at lower energies, $E_{\text {beam }} \approx 0.2-5 \mathrm{~A} \cdot \mathrm{GeV}$, one finds that $T_{f o}$ decreases dramatically as the beam energy decreases. This observation implies that the freeze-out temperature $T_{f o}$ saturates around $150 \mathrm{MeV}$ at a beam energy of about $10 \mathrm{GeV}$ as already discussed in ref. $[2,3,17]$.

\subsection{Particle Ratios}

Having fixed the freeze-out temperature parameter $T_{f o}$, we now turn to the issue of chemical potentials. Assuming chemical equilibrium, this information can be extracted from the measured particle ratios. The NA44 spectrometer can measure particles of both signs at the same $p_{T}$ and $y$ window. It is therefore an unique advantage for studying the particle production ratios since many systematic errors will cancel out. In Fig. 3, the preliminary $\mathrm{K}^{-} / \mathrm{K}^{+}$and $\overline{\mathrm{p}} / \mathrm{p}$ ratios are shown as a function of rapidity for both $\mathrm{S}+\mathrm{S}$ and $\mathrm{Pb}+\mathrm{Pb}$ central collisions. Model predictions ${ }^{4}$ of RQMD(v1.08) [18] and VENUS(v4.12) [19] are represented by dashed and solid lines, respectively. The systematic errors on the ratios for $\mathrm{Pb}+\mathrm{Pb}$ collisions are

\footnotetext{
${ }^{4}$ The calculations were performed with the similar centrality cut as done in the experiment.
} 
estimated to be about $15 \%$. The main contributors to the rather large error bars are centrality determination and pion-veto corrections. For the $\mathrm{S}+\mathrm{S}$ collisions, the systematic errors are about $11 \%$.

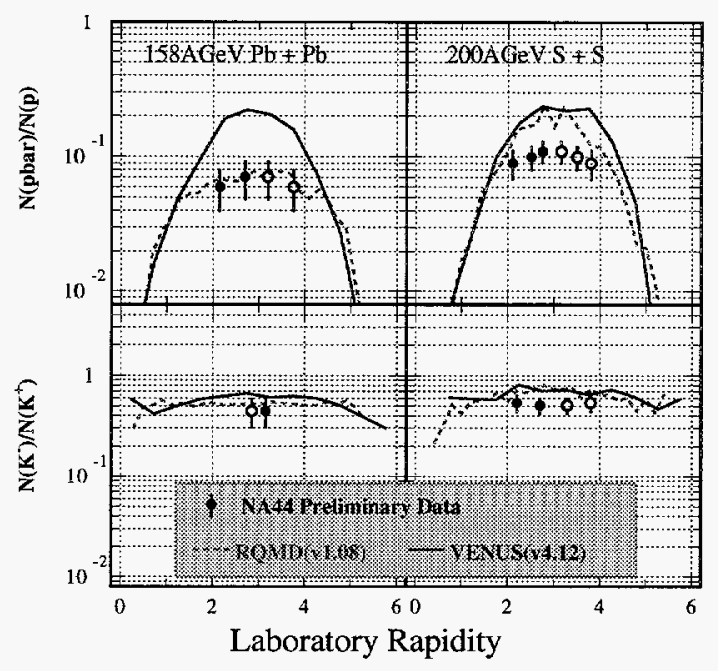

Figure 3. NA44 preliminary $K^{-} / K^{+}$and $\bar{p} / p \quad r a-$ tios. Open circles are mirrored about the central rapidity of 3.0 and 2.9 for $S+S$ and $\mathrm{Pb}+\mathrm{Pb}$ central collisions, respectively. $R Q M D(v 1.08)$ and VENUS(v4.12) predictions are shown in dashed and solid lines.

In case of $\overline{\mathrm{p}}$ to $\mathrm{p}$ ratio, both model predictions deviate from the data by a factor of 3 for the light colliding system $\mathrm{S}+\mathrm{S}$. For the $\mathrm{Pb}+\mathrm{Pb}$ collisions, RQMD seems to give a better results. However, for kaons, both model always overpredict the ratios. It is clear here that much work has to be done in order to understand the details of the particle production in heavy-ion collisions.

Particle density ratios determine the quantity: $\mu_{i} / T_{f o}^{c h}{ }^{5}$ At the quark level, there are two chemical potentials $\mu_{q}$ and $\mu_{s}$ for light and strange quarks, respectively. The relationship between the bayonic and quark chemical potentials can be found in several references, for example, ref. [7, 21]. Assuming the condition of chemical equilibrium, the values of $\left(\mu_{q}, \mu_{s}\right)$ can be extracted from the measured particle ratios. The results of this analysis from the $\bar{p} / \mathrm{p}$ and $K^{-} / K^{+}$ratios around mid-rapidity are listed in Table I.

Table I. Light and strang quark chemical potentials $\left(\mu_{q}, \mu_{s}\right)$ extracted from $S+S$ and $\mathrm{Pb}+\mathrm{Pb}$ central collisions. (All results are obtained from the mid-rapidity region.)

\begin{tabular}{||c||c|c||}
\hline \hline & $\mu_{q}(\mathrm{MeV})$ & $\mu_{s}(\mathrm{MeV})$ \\
\hline $200 \mathrm{~A} \cdot \mathrm{GeV} \mathrm{S}+\mathrm{S}$ & $53 \pm 8$ & $\sim 0$ \\
\hline $158 \mathrm{~A} \cdot \mathrm{GeV} \mathrm{Pb}+\mathrm{Pb}$ & $71 \pm 15$ & $5 \pm 2$ \\
\hline
\end{tabular}

Within the model considered here, the results are not dependent on how one

\footnotetext{
${ }^{5}$ Here $T_{f \circ}^{c h}$ is the chemical freeze-out temperature parameter which is determined by the particle ratio study. See below for more detailed discussion.
} 
treats the collective flow because only particle ratios are used. On the one hand, within the experimental error bars, the values of the chemical potentials are consistent with the values found in ref. [5, 22], even though particle mass up to $2 \mathrm{GeV}$ and strange quantum number of $\mathrm{S}=2$ were included in those studies. This may simply reflect the fact that once equilibrium is established, a subset of the data is sufficient to extract the thermal parameters. On the other hand, from the ratio analysis of $200 \mathrm{~A} \cdot \mathrm{GeV} / \mathrm{c}$ S-beam induced collisions $\mathrm{S}+\mathrm{Pb}(\mathrm{W})$, the chemical freezeout temperature parameter $T_{f o}^{c h}$ is found consistently higher than $150 \mathrm{MeV}$, i.e., $T_{f o}^{c h} \approx 170 \sim 200 \mathrm{MeV}$. Since the fireball produced in the heavy-ion collisions is an open thermodynamic system and the inelastic process requires more energy than elastic process, it is conceivable that the chemical freeze-out may occur earlier than thermal one [21].

Because of their large interaction cross section, antiprotons provide a unique probe of the hadronic environment. By studying the antiproton distribution, one gains information on dynamic processes such as pair production, annihilation, and mean-field-effects of the fireball. As has been noted by Gavin et al. [23] the $\bar{p} / \mathrm{p}$ ratios are useful to understand the spacetime evolution of baryons in heavy-ion collisions. Experiment NA44 $[24,25]$ has measured proton and antiproton spectra with high statistics and has compared results of $\mathrm{p}+\mathrm{p}, \mathrm{p}+\mathrm{A}$, and $\mathrm{A}+\mathrm{A}$ collisions in the same spectrometer. Fig. 4 shows the mid-rapidity $\bar{p} / \mathrm{p}$ ratios all the way from the elementary collision $\mathrm{p}+\mathrm{p}$ to the truly heavy-ion $\mathrm{Pb}+\mathrm{Pb}$ collisions.

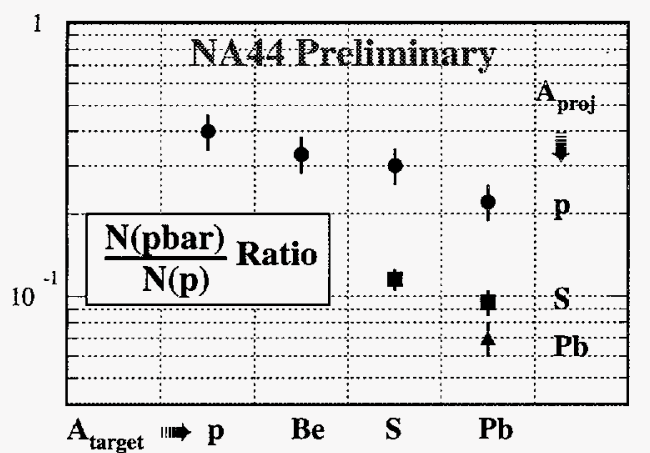

Figure 4. NA44 preliminary $\overline{\mathrm{p}}$ to $p$ ratios for several projectile and target combinations. $p$ results is taken from ref. [14].

In high energy $\mathrm{p}+\mathrm{p}$ collisions [20], pair creation is the largest source of central $\mathrm{p}$ and $\overline{\mathrm{p}}$. This implies that antiprotons are always produced with accompanying protons. In the case of heavy-ion collisions where a large number of protons are also shifted from the target and projectile into the mid-rapidity region, the $\bar{p} / \mathrm{p}$ ratio will further decrease compared to that of proton induced collisions. This is consistent with the observed systematic trends. However, the measured ratios are the combined results of particle production at the earlier collision stage and later annihilation. 


\section{Summary}

We have discussed the results of mid-rapidity transverse momentum distributions of pions, kaons, and protons from $\mathrm{p}+\mathrm{p}, \mathrm{S}+\mathrm{S}$ and $\mathrm{Pb}+\mathrm{Pb}$ central collisions. The systematics of the data strongly suggests a freeze-out temperature of $T_{f o} \approx 145 \pm 15$ $\mathrm{MeV}$ for collisions above beam energy $\sim 10 \mathrm{~A} \cdot \mathrm{GeV}$. Current results of cascade models RQMD(v1.08) and VENUS(v4.12) cannot fully reproduce the observed particle ratios. Systematic results of $\bar{p} / p$ ratios, from $p+p$ to $\mathrm{Pb}+\mathrm{Pb}$ central collisions, studied with the NA44 spectrometer were also presented. Such long awaited information should help to understand the collision dynamics.

All of our discussions were based on the measured light hadrons at the freezeout stage. If thermal and chemical equilibria are indeed reached, information on the earlier stages of the collision is lost. In order to get insight into the initial condition of heavy ion collisions, we need to investigate the distributions and yields of leptons and photons and possibly high mass multi-strange resonances.

\section{Acknowledgement}

The author wishes to express his thanks to professors J.Rafelski and A.D. Panagiotou for many helpful discussions on the physics of chemical equilibrium in heavyion collisions. This work is supported by the US Department of Energy. We are grateful to the staff of the CERN PS-SPS accelerator complex for their excellent work. We thank the technical staff at CERN and the collaborating institutes for their valuable contribution. We are also grateful for the support given by the Austrian Fonds zur Foerderung der Wissenschaftlichen Forschung; the Science Research Council of Denmark; the Japanese Society for the Promotion of Science; the Ministry of Education, Science and Culture, Japan; the Science Research Council of Sweden; the National Science Foundation.

\section{References}

1. E. Fermi, Progr. Theor. Phys. 5, 570(1950).

2. I.Ya. Pomeranchuk, Dokl. Akad. Sci. U.S.S.R. 78, 884(1951).

3. L.D.Landau, Izv. Akad. Nuak SSSR Ser. Fiz. 17, 51(1953).

4. E. Schnedermann, Y. Sollfrank, and U. Heinz, Phys. Rev. C48, 2462(1993).

5. P. Braun-Munzinger, J. Stachel, J.P. Wessels, and N. Xu, Phys. Lett. B344, 43(1995); ibid, B365,1(1996).

6. J. Letessier, J. Rafelski, and A. Tounsi, Phys. Lett. B328, 499(1994) and J. Letessier, A. Tounsi, U. Heinz, J. Sollfrank, and J. Rafelski, Phys. Rev. Lett., 70, 3530(1993).

7. P. Braun-Munzinger and J. Stachel, J. Phys. G: Nucl. Part. Phys., 21, L17(1995). 
8. V. Koch, G.E. Brown, and C.M. Ko, Phys. Lett. B265, 29(1991); V. Koch, Nucl. Phys. A591, 531c(1995).

9. H. Beker et al., (The NA44 Collaboration), Phys. Lett. B302, 510(1993).

10. A. Franz, (The NA44 Collaboration), Quark Matter '96 Proceedings, May 1996.

11. N. Xu, (The NA44 Collaboration), Quark Matter '96 Proceedings, May 1996, and H. Bøggiled, The NA 44 Collaboration, to be submitted to Phys. Lett. B.

12. H. Bøggiled et al., (The NA44 Collaboration), Phys. Lett. B, in press.

13. J. Pluta et al., using RQMD plus longitudinal comoving charge of $Z_{\text {eff }}=40$ per unit rapidity, the observed $\mathrm{N}\left(\pi^{-}\right) / \mathrm{N}\left(\pi^{+}\right)$ratio $[11,12]$ can be well reproduced.

14. B. Alper et al., Nucl. Phys. B100, 237(1975) and Guettler et al., Nucl. Phys. B116, 77(76).

15. F. Cooper, G. Frye, and E. Schonberg, Phys. Rev. Lett. 32, 862(1974).

16. E.V. Shuryak, Sov. J. Nucl. Phys., V20, No.3, 295(1972).

17. R. Hagedorn, Suppl. Nuovo Cim. III.2, 147(1965); H. Stöcker, A.A. Ogloblin, and W. Greiner, Z. Phys. A303, 259(1981).

18. H. Sorge, H. Stöcker, and W. Greiner, Ann. Phys. (NY), 192, 266(1989); H. Sorge, Phys. Rev. C52, 3291(1995).

19. K. Werner, Phys. Rep. 232, 87(1993).

20. L. Camilleri, Phys. Rep. V144(2), 51(1987).

21. J. Letessier et al., Phys. Rev. C51, 3408(1995).

22. J. Rafelski and M. Danos, Phys. Rev. C50, 1684(1994).

23. S. Gavin, M. Gyulassy, M. Plümer and R. Venugopalan, Phys. Lett. B234, 175(1990).

24. B.V. Jacak, NATO Advance Study Institute, 'Hot and Dense Nuclear Matter', p607, Bodrum, Turkey, Ed. W. Greiner, H. Stočker, and A. Gallmann, Plenum Press, 1994.

25. M. Murray, (The NA44 Collaboration), Proceedings of 'Strangeness in Hadronic Matter', p162, Ed. J. Rafelski, AIP Press, 1995. 